Trauma Berufskrankh 2016 · [Suppl 2]: 18:S105-S110

DOI 10.1007/s10039-015-0063-z

Online publiziert: 1. September 2015

๑) Springer-Verlag Berlin Heidelberg 2015

CrossMark

Thomas Lein

Klinik für Unfallchirurgie, Diakonissenkrankenhaus Dresden, Dresden, Deutschland

\title{
Kreuzbandverletzungen bei offenen Wachstumsfugen
}

\section{Konservative Therapie}

zung des vorderen Kreuzbandes (VKB) liegen zum Unfallzeitpunkt noch offene Wachstumsfugen vor [29]. Die jährliche Inzidenz für eine Kreuzbandverletzung bei Kindern und Jugendlichen wird mit 1/100.000 beziffert und stellt daher ein nicht ganz so häufiges Ereignis beim Adoleszenten dar (• Abb. 1). Allerdings kann auch davon ausgegangen werden, dass bei einem traumatischen Hämarthros des Kniegelenks ohne erfolgte Patellaluxation in bis zu $65 \%$ der Fälle das VKB mitbeteiligt ist [26]. Nach Diagnosesicherung einer Kreuzbandruptur beim Kind stellte sich hier in der Vergangenheit die Frage der weiteren Therapieentscheidung. Während 2006 in einer Umfrage der Anterior Cruciate Ligament Study Group bei einem 8 -jährigen Jungen noch $58 \%$ der Befragten konservativ behandeln wollten, hat sich in jüngerer Vergangenheit ein Umdenken eingestellt, d. h. die operative Versorgung von Kreuzbandverletzungen im Wachstumsalter wird zunehmend empfohlen.

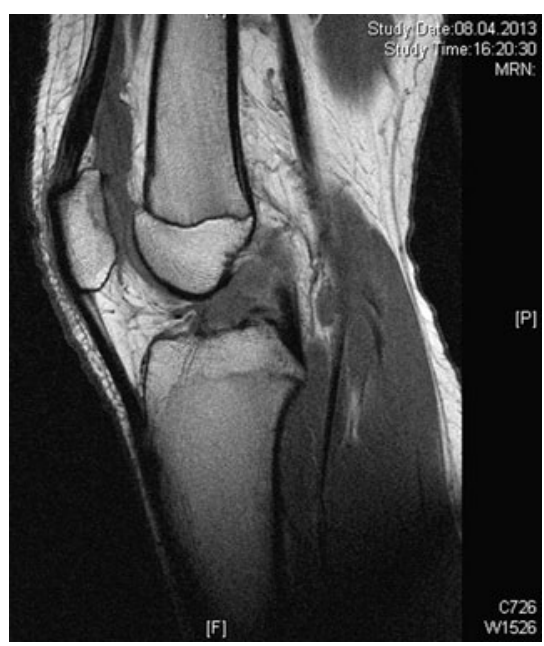

Abb. $1 \Delta$ Magnetresonanztomogramm einer 13-jährigen Patientin mit vollständiger Ruptur des vorderen Kreuzbandes beim Schulsport
Die konservative Therapie war bis weit in die 1990er Jahre die favorisierte Vorgehensweise, da durch das operative Vorgehen eine Verletzung der noch offenen Wachstumsfuge mit der Folge eines konsekutiven Fehlwachstums oder einer Wachstumsstörung befürchtet wurde. Die Therapie schloss eine restriktive Behandlung mit konsequenter Orthesenanlage, Verbot für alle „pivoting“ Sportarten und intermittierender Physiotherapie zum Muskelaufbau ein. Allerdings mehrten sich mit den Jahren Hinweise darauf, dass insbesondere bei Kindern die Compliance für ein derartiges Vorgehen zu hinterfragen ist, da diese hochaktiv sind und die Instabilität nicht ausreichend wahrnehmen. Untersuchungen deuteten auf damit verbundene Spätschäden nach konservativer Versorgung hin. So wurde gezeigt, dass nach 3 Jahren in $54 \%$ der Fälle schlechte Ergebnisse vorlagen [1], es in $50-75 \% \mathrm{zu}$ sekundären Knorpel- und Meniskusschä-

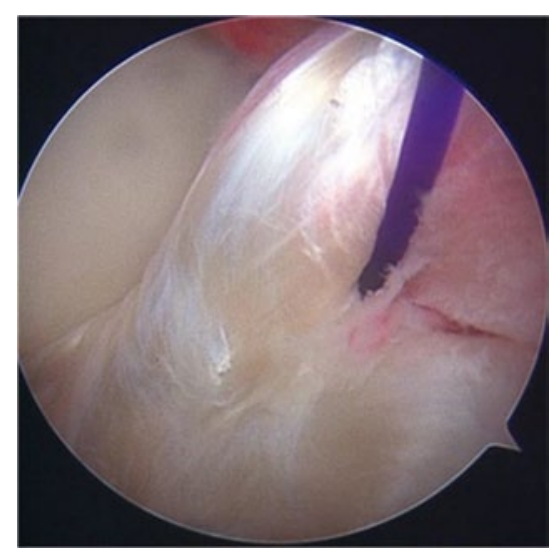

Abb. 2 A Intraoperatives arthroskopisches Bild: Versuch einer proximalen Refixation und Naht des vorderen Kreuzbandes den kam $[4,12,27]$ und in bis zu 90\% der Fälle eine Instabilität resultierte [21]. Auch ist die Rate der Patienten hinsichtlich der Wiederaufnahme einer vorbestehenden sportlichen Aktivität mit 5-41\% eher als gering anzusehen [22].

In einer systematischen Analyse von 60 Studien konnten Preiss et al. [20] aufzeigen, dass die operative Vorgehensweise bei der Ruptur des VKB offenbar deutliche Vorteile gegenüber einer konservativen Therapie hat. Die Ergebnisse belegen, dass hinsichtlich des Langzeiterfolgs offenbar ein Großteil der Patienten mit VKB-Ruptur im Wachstumsalter von einer operativen Therapie profitiert [2].

\section{Naht des vorderen Kreuzbands}

In der Vergangenheit wurde immer wieder versucht, durch Naht des VKB in Verbindung mit einer transossären Fixation der Gefahr einer Fugenschädigung auszuweichen (• Abb. 2). Allerdings zeigt diese Technik häufig wenig vorhersagba-

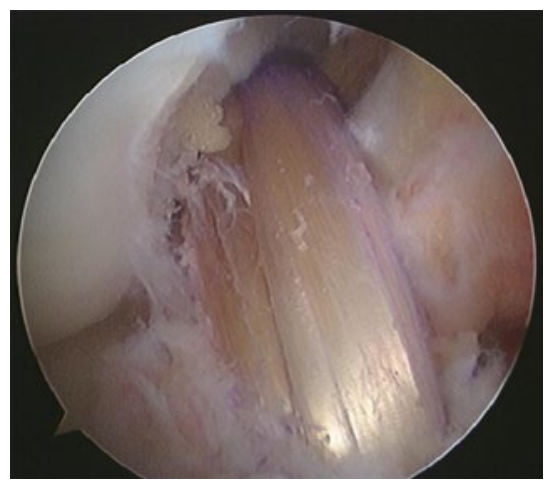

Abb. 3 A Intraoperatives arthroskopisches Bild: Vierfachtransplantat bei einem 14-jährigen Patienten. Femorale Bohrung anatomisch über den anteromedialen Arbeitszugang 


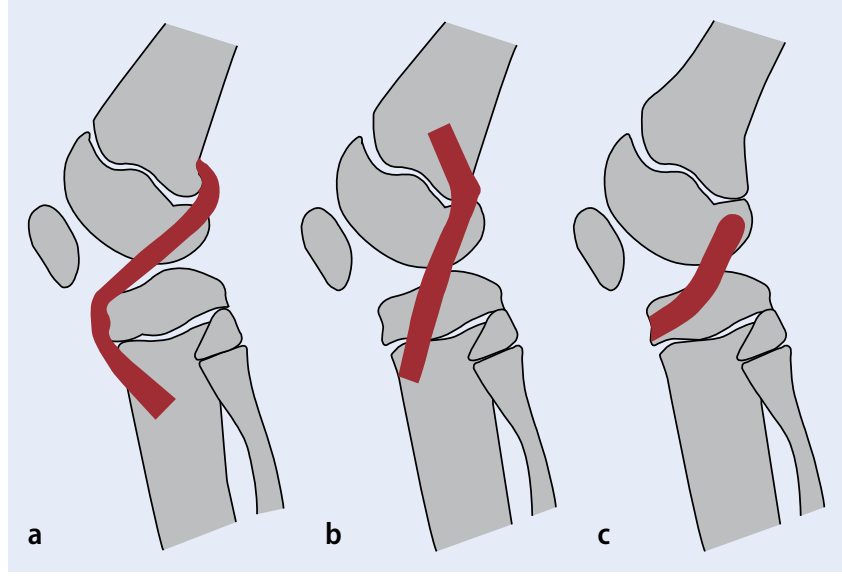

Abb. $4<$ Möglichkeiten der Transplantateinbringung bei Patienten mit offenen Wachstumsfugen: a extraepiphysär, b fugenkreuzend oder c rein epiphysär
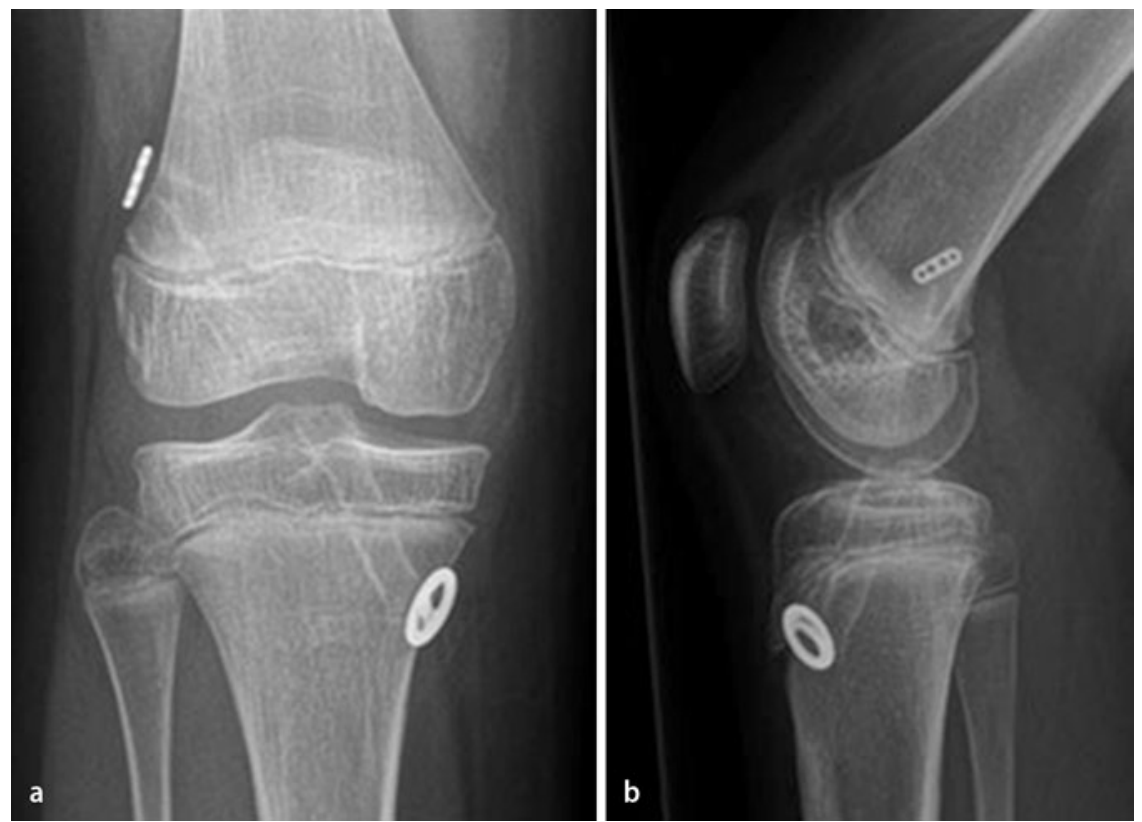

Abb. $5 \Delta$ a,b Röntgenkontrolle. Gelenkferne Fixation des Transplantats über Endobutton und „suture disc"

re gute Ergebnisse. In einer Metaanalyse von Frosch et al. [3] lag der Lysholm-Score bei unter 80 Punkten und im International Knee Documentation Committee (IKDC-)Score erreichten nur knapp 40\% der Patienten Grad A und B; demgegenüber waren nach Kreuzbandrekonstruktion bei mehr als $80 \%$ gute und sehr gute Ergebnisse zu konstatieren. Daher kann die Kreuzbandnaht in transossärer Technik als Alternative zum Kreuzbandersatz derzeit nicht als generelle Therapie empfohlen werden. Eine Ausnahme für eine „healing response“ mögen hier elefantenfußartige proximale Ausrisse oder Partialrupturen des VKB darstellen, ggf. in Verbindung mit Mikrofrakturieren der lateralen Femurwange.
Aufgrund der aktuellen Datenlage ist davon auszugehen, dass ein Großteil der Verletzungen des VKB bei offenen Fugen mit einer Kreuzbandrekonstruktion mittels Sehnenplastik versorgt werden sollte.

\section{Transplantatwahl zur Rekonstruktion}

Bei Kindern und Jugendlichen mit offenen Wachstumsfugen stehen prinzipiell die gleichen Sehnentransplantate zur Verfügung wie beim Erwachsenen. In der Vergangenheit wurde eine Reihe von möglichen autologen Transplantaten zum Kreuzbandersatz vorgestellt, von Fascia-lata-Streifen über Patellar- und Quadrizepssehne bis hin zu den "hamstrings“.
Im eigenen Vorgehen bevorzugen wir bei allen kindlichen intraligamentären VKB-Rupturen die operative Therapie mittels Ersatzplastik mit autologem Semitendinosustransplantat. Je nach Transplantatlänge und -durchmesser kann zwischen einem Zwei-, Drei- oder Vierfachtransplantat (• Abb. 3) gewählt werden, u. U. muss bei schmächtigen Transplantaten zusätzlich die Gracilissehne zur Anwendung kommen. Dabei ermöglicht die indirekte femorale Fixation z. B. unter Verwendung eines Endobuttons eine kürzere Transplantatlänge, wobei der femorale Bohrkanal eine Länge von $15 \mathrm{~mm}$ nicht unterschreiten sollte, um eine sichere Einheilung im Verlauf nicht zu gefährden.

Das mittlere Patellarsehnendrittel als Knochen-Sehne-Knochen- oder PeriostSehne-Periost-Transplantat in Alternative zu den autologen Sehnentransplantaten wurde in der Vergangenheit zunehmend seltener eingesetzt $[6,8,24]$. Bei Verwendung der Patellarsehne sollte unbedingt darauf geachtet werden, dass die Fixation der endständigen Knochenblöcke außerhalb der Fugen erfolgen muss, um hier einen vorzeitigen Fugenverschluss zu vermeiden. Im eigenen klinischen Alltag benutzen wir diese allenfalls als Revisionstransplantat bei der Reruptur.

\section{Hinweise zur Operationstechnik}

Hinsichtlich der Transplantatplatzierung kann zwischen den extra- und rein epiphysealen Techniken sowie der wachstumsfugenübergreifenden transphysären Vorgehensweise gewählt werden (- Abb. 4). Preiss et al. [2, 20] konnten zeigen, dass die fugenkreuzende Technik bei exakter Ausführung offensichtlich bessere Ergebnisse hinsichtlich einer späteren Achsenabweichung und Längendifferenz aufweist als die fugenschonenden Verfahren. Die in der Literatur beschriebenen Wachstumsstörungen bei transepiphysärem Vorgehen scheinen i.d.R. durch fehlerhafte Technik und Minderbeachtung der Verletzlichkeit der Epiphysenfugen begründet [6]. Hauptursachen waren falsch angelegte Bohrkanäle, fugenkreuzende Knochenblöcke oder in der Wachstumsfuge liegende Fixationselemente. Generell wird die Gefahr der Fugenschädigung mit relevanten Folgen of- 
fenbar überschätzt. So konnten Kercher et al. [5] aufzeigen, dass ein Bohrkanal von $8 \mathrm{~mm}$ bei exakter Vorgehensweise eine Fugenschädigung von nur bis zu $3 \%$ hervorruft. Darüber hinaus finden sich in der Literatur Hinweise, dass eine Schädigung des mediolateralen Durchmessers von bis zu $20 \%$ der Fuge toleriert wird $[9,15]$.

\section{Anlage des tibialen Bohrkanals}

Bei der exakten Anlage des tibialen Tunnels gilt es eine Reihe von Besonderheiten zu beachten. Um die verletzliche Apophyse der Tuberositas tibiae zu schonen, sollte das Zielgerät auf $55-60^{\circ}$ eingestellt werden. Damit lässt sich eine sichere Lage mit steiler und fast senkrechter Position unter möglichst zentraler Perforation der Fuge erreichen [28]. Der tibiale Bohrkanal sollte an der Grenze vom 2. zum 3. Quadranten nach Harner liegen [23]. Um einer Hitzeschädigung vorzubeugen, muss niedertourig und schonend gebohrt werden, manche Autoren verweisen auf die Verwendung eines Handbohrers. Der Transplantatdurchmesser sollte möglichst klein gewählt werden, eine gute Kanalfüllung durch das Transplantat ist anzustreben („Press-fit“-Lage). Allerdings ist die Impaktion des Bohrkanals durch Dilatatoren unbedingt $\mathrm{zu}$ vermeiden, um hier keine zusätzliche Traumatisierung der Fuge hervorzurufen. In der Regel sind Transplantate mit einem Durchmesser von 6-8 mm anzustreben, sie reichen für die zu erwartende Stabilität aus.

\section{Anlage des femoralen Bohrkanals}

Ein zu steil angelegter femoraler Tunnel in „High-Noon-Position“ hat Nachteile hinsichtlich der zu erreichenden Rotationsstabilität, daher ist die transtibiale Anlage nur bei Kindern mit weit offenen Fugen und hohem Wachstumspotenzial zu empfehlen. Durch die Bohrung über das anteromediale Portal lässt sich i.d.R. eine mehr anatomische Kanallage femoral erreichen, allerdings ist durch den schrägeren Verlauf auch eine höhere Gefahr der Fugenverletzung gegeben. Insbesondere die Verletzung des peripher gelegenen Ranvier-Schnürrings oder des perichondralen Rings nach LaCroix kann Ursache für eine asymmetrische Wachstumsstö-

Trauma Berufskrankh 2016 · [Suppl 2]: 18:S105-S110 DOI 10.1007/s10039-015-0063-z

(c) Springer-Verlag Berlin Heidelberg 2015

\section{T. Lein}

\section{Kreuzbandverletzungen bei offenen Wachstumsfugen}

\section{Zusammenfassung}

Die chirurgische Behandlungsoption bei Verletzungen des vorderen Kreuzbandes (VKB) bei noch offenen Wachstumsfugen hat sich in den vergangenen Jahren deutlich geändert. Während bei der intraligamentären Ruptur die Indikation zum Kreuzbandersatz in der Vergangenheit noch sehr zurückhaltend gestellt wurde, war die Indikation zur operativen Behandlung ossärer Ausrisse der Eminentia intercondylaris klarer definiert. Die anatomische Refixation des knöchernen Anteils führt hier zu guten bis sehr guten Ergebnissen. Die Behandlung intraligamentärer Rup- turen des VKB bei offenen Wachstumsfugen wird noch kontrovers diskutiert, es zeigt sich jedoch ein Trend zur operativen Versorgung. In diesem Beitrag wird aufgezeigt, dass bei exakter Vorgehensweise relevante Verletzungen der Wachstumsfuge selten vorkommen und häufig auf technische Fehler zurückzuführen sind.

Schlüsselwörter Kinder · Vorderes Kreuzband . Wachstumsfuge · Sehnentransfer . Wachstumsstörungen

\section{Rupture of the anterior cruciate ligament with open growth plates}

\section{Abstract}

The surgical procedure for injuries of the anterior cruciate ligament $(\mathrm{ACL})$ with an open epiphyseal growth plate has fundamentally changed in recent years. Although the indications for surgical reconstruction of the $\mathrm{ACL}$ after intraligamental rupture were in the past very reserved, the indications for operative treatment of osseous avulsion of the eminences were more clearly defined. The anatomically correct fixation of the avulsed osseous fragment leads to good up to very good results. The management of intraligamental tears of the $A C L$ in cases of an open growth plate is still under discussion but in the literature there is a general trend towards operative treatment. In this article it is shown that relevant injuries of the growth plate are rare when an exact approach is used and are often related to technical failures.

\section{Keywords}

Children - Anterior cruciate ligament - Growth plate - Tendon transfer - Growth disorders rung sein [19]. Daher sollte ein Ausbrechen der dorsalen Femurkortikalis zwingend vermieden und auf ein Aufraspeln der dorsalen Kortikalis verzichtet werden. Um eine exakte Tunnellage zu erreichen, empfiehlt sich zudem der Einsatz eines femoralen Zielgeräts mit 5-7 mm Offset in der „Over-the-Top“-Position je nach Größe des Kniegelenks und des gewonnenen Transplantats. Im arthroskopischen Bild sollte die Wachstumsfuge im Kanal kreisrund und nicht ovalär zur Darstellung kommen und keine Unterbrechung aufweisen.

\section{Einzug und Sehnenfixation}

Das Transplantat sollte mit leichtem Widerstand in das Gelenk gezogen werden, um eine annähernde „Press-fit“-Situation zu erreichen. Das Gelenk wird unter moderater Vorspannung mehrfach durchbe- wegt und auf Isometrie und Notch-Impingement kontrolliert. Ein zu straffes Einspannen des Transplantats sollte wegen der Gefahr eines Epiphyseodeseeffekts sorgfältig gemieden werden.

Bei Jugendlichen im Wachstumsstadium IV nach Tanner kann die Fixation wie beim Erwachsenen ohne Einschränkung erfolgen, auch die direkte Fixation mit Interferenzschrauben lässt hier keine Wachstumsstörung im Verlauf erwarten. Bei Kindern mit Wachstumsfugen bis Stadium III nach Tanner empfiehlt sich eine femorale extrakortikale indirekte Fixation mit Kippknöpfen oder Endobutton. Die femorale Fixation kann direkt oder indirekt erfolgen, jedoch muss darauf geachtet werden, dass keine fugenüberbrückenden Transplantate (Schrauben, Klammern etc.) resultieren, da diese mit Sicherheit zur Störung der Wachstumsfuge im Verlauf führen [14]. Die Länge von Bohrka- 


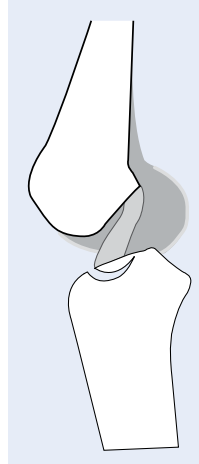

Typ 1

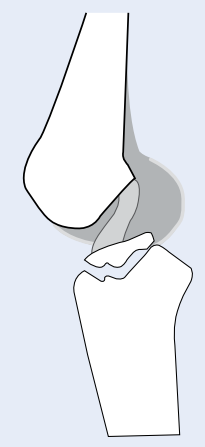

Typ 3b

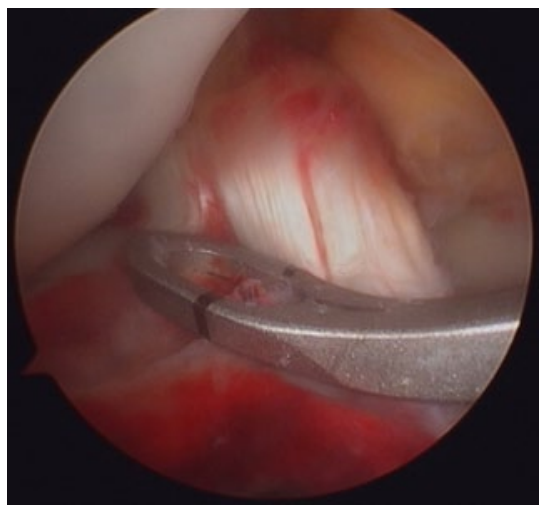

Abb. $8 \Delta$ Arthroskopische Fixation des Avulsionsfragments mit Kirschner-Drähten transtibial unter Verwendung des Kreuzbandzielinstrumentariums

naleingang bis Wachstumsfuge kann arthroskopisch mit einem skalierten Messdraht ermittelt werden. Sollte die Sehnenfixation auf diesem Weg nicht möglich sein, kann auch eine indirekte tibiale Fixation mit Titanknöpfen oder-schrauben erfolgen, was insbesondere bei sehr jungen Patienten zu empfehlen ist (• Abb.5). Die gelenkferne Fixation erhöht dabei die Rerupturrate nach Datenlage offenbar nicht [29].

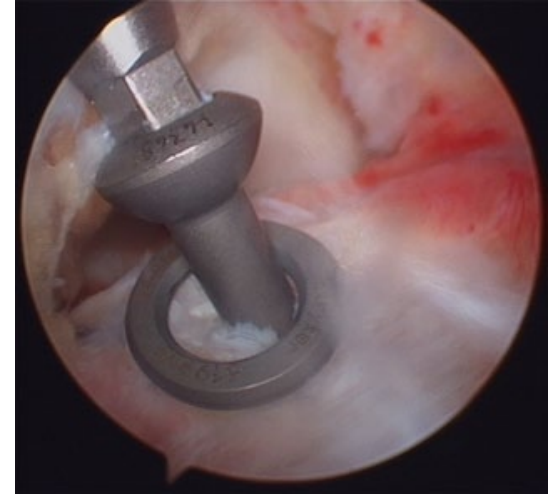

Abb. $9 \Delta$ Arthroskopische Verschraubung eines Eminentiafragments (knöcherner Ansatz des vorderen Kreuzbandes) mit kleiner Lochschraube samt Unterlegscheibe

\section{Fraktur der Eminentia intercondylaris}

\section{Häufigkeit und Klassifikation}

Die Frakturen der Eminentia intercondylaris treten im nichtartikulären Bereich des Tibiaplateaus auf und entsprechen dem knöchernen Ausriss des VKB im Sinne einer Avulsionsverletzung. Sie sind bedingt durch die bei Kindern höhere biomechanische Stabilität des Kreuzbandes im Vergleich zur osteochondralen Verankerung. Diese Verletzung findet sich am

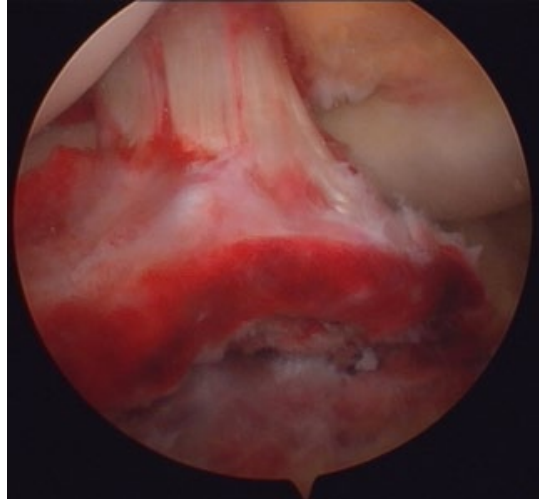

Abb. 7 Intraoperatives arthroskopisches Bild: Eminentiaausriss Typ III nach Säuberung und Grobreposition. Die knöcherne Befestigung des vorderen Kreuzbandes war völlig desinseriert

häufigsten bei Heranwachsenden im Alter von 8 bis 14 Jahren, die Inzidenz wird mit 3 Fällen pro 100.000 Einwohner im Jahr angegeben $[7,17]$.

Bereits 1959 wurden die Eminentiafrakturen von Meyers u. McKeever klassifiziert [11] und später von Zaricznyj [30] durch den Typ 4 erweitert. Typ 1 zeigt keine Dislokation, beim Typ 2 ist der ventrale Anteil der Eminentia disloziert, der Typ 3a beschränkt sich auf die Dislokation der Kreuzbandinsertion, während beim Typ 3b die gesamte Eminentia betroffen ist. Beim Typ 4 liegen rotierte oder multifokale Knochenfragmente vor (• Abb. 6).

\section{Therapie}

Ziel der Behandlung ist der Erhalt der anterioren Stabilität des Kniegelenks. Nichtdislozierte Frakturen werden übereinstimmend konservativ in einer Knieorthese für 6 Wochen in einer Flexionsstellung von $10^{\circ}$ unter Vollbelastung immobilisiert. Auch die Typ-2-Ausrisse können konservativ behandelt werden, allerdings kann im Verlauf auch eine Sekundärdislokation des Fragments eintreten, sodass auch hier eine stabile Retention erfolgen sollte. Zudem besteht bei diesem Typ zusätzlich die Gefahr einer Heilungsstörung, wenn der ventrale Anteil über dem Ligamentum genus transversum zu liegen kommt und dieses einklemmt. Frakturen vom Typ 3 und 4 stellen eine absolute Indikation zur operativen Revision, Reposition und Fragmentfixation dar. Dabei erscheint es für den Langzeitverlauf unerheblich, ob hier eine Miniarthrotomie 

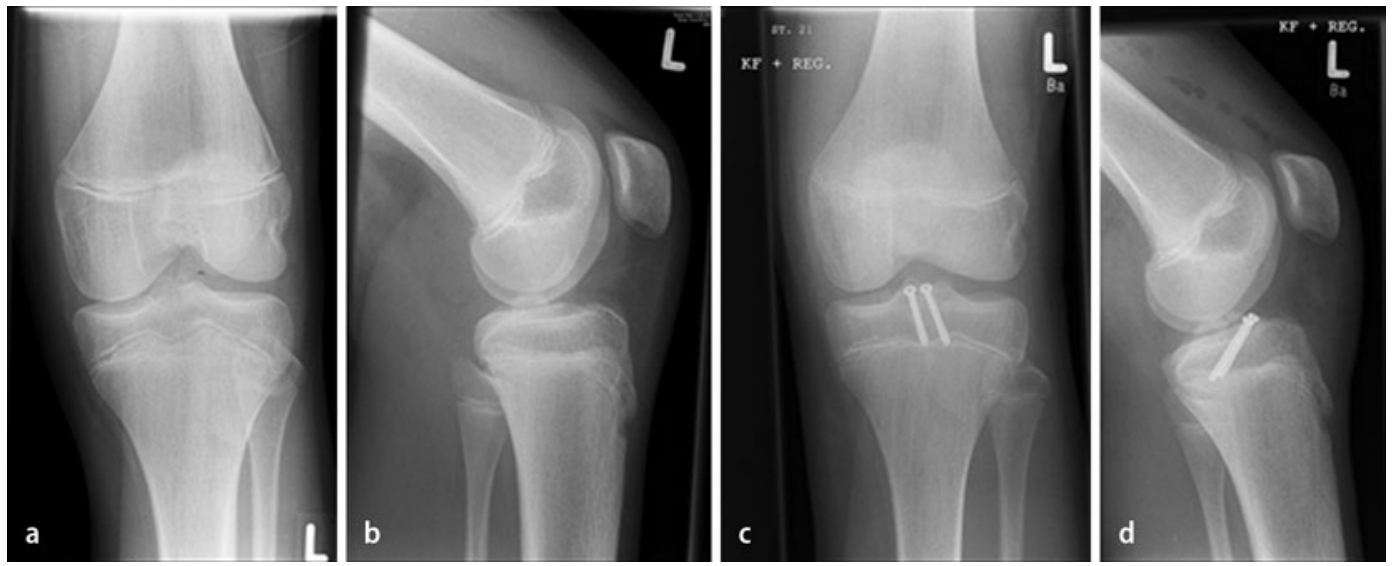

Abb. $10<$ a,b Radiologische Darstellung einer Eminentiafraktur nach Zweikampf beim Fußball bei einem 13-jährigen Patienten. c,d Postoperatives Ergebnis nach direkter Verschraubung, die proximale Epiphysenfuge an der Tibia wurde geschont

durchgeführt oder die rein arthroskopische Vorgehensweise gewählt wird; wichtig ist, dass das Fragment anatomisch reponiert und stabil fixiert wird $[10,17]$.

Hinsichtlich der Vorgehensweise muss das Eminentiafragment aufgesucht, gesäubert und eingepasst werden (• Abb. 7). Danach kann die temporäre oder definitive Fixation mit direkt oder indirekt eingebrachten Kirschner-Drähten erfolgen ( $\bullet$ Abb. 8). Für die Fixation steht eine Reihe von verschiedenen Techniken und Möglichkeiten zur Verfügung. Bei größeren Ausrissfragmenten können Lochschrauben über einen hohen Zugang direkt oder unter Zuhilfenahme eines Zielinstruments indirekt eingebracht werden, wobei sich die Verwendung einer kleinen Unterlegscheibe empfiehlt (• Abb. 9, [16]). Das Fragment sollte ungefähr die 2- bis 3-fache Größe des Schraubendurchmessers haben, damit es sich sicher fixieren lässt, auch kann das Einbringen von 2 kleineren Schrauben zur Rotationssicherheit beitragen (- Abb. 10) Bei kleineren oder multiplen Frakturen bieten sich gekreuzte Kirschner-Drähte [25], Fadentechniken oder aber Kippanker an, wie u. a. von Pape u. Griffin beschrieben [18].

\section{Nachbehandlung}

Sowohl nach Kreuzbandersatz als auch nach Refixation bei Eminentiaausriss empfiehlt sich die postoperative Ruhigstellung in einer kindgerechten Knieorthese im Bewegungsausmaß Extension/ Flexion $0 / 0 / 90^{\circ}$ für 6 Wochen. In den ersten beiden Wochen nach der Operation sollte dabei ein Abrollkontakt an Unter- armgehstützen mit Teilbelastung des betroffenen Beins von $20 \mathrm{~kg}$ erfolgen. Erst danach ist der Übergang zur schmerzadaptierten Vollbelastung gestattet, um ein "Reizknie“ zu vermeiden. In der Regel sollte bei Kindern die Rehabilitation nach Kreuzbandoperation strenger als beim Erwachsenen erfolgen, was der zu erwartenden Compliance und dem Bewegungsdrang der jungen Patienten geschuldet ist. Daher erscheinen engmaschigere klinische Kontrollen bei dieser Patientengruppe angezeigt. Hinsichtlich des Einheilungsverhaltens im Wachstum führt die viskoelastische Deformierung („creep“) zur Längenanpassung unter konstant haltender Last. Es kann davon ausgegangen werden, dass das Phänomen BelastungEntspannung (,stress relaxation“) unter konstanter Deformation zur Abnahme von Stress im Gewebe führt [13].

\section{Fazit für die Praxis}

- Nach aktueller Datenlage sollte die Indikation zur Ersatzoperation des vorderen Kreuzbandes bei Kindern und Heranwachsenden großzügig gestellt werden, da die Ergebnisse nach konservativer Therapie und Kreuzbandnaht unbefriedigend waren und das Risiko von Sekundärschäden als hoch einzuschätzen ist.

- Bei exakter Ausführung mit korrekter Tunnellage ist die fugenübergreifende transepiphysäre Technik ein sicheres Verfahren.

- Postoperative Wachstumsstörungen sind nur in bis etwa $2 \%$ der Fälle zu erwarten, wenn die Verletzlichkeit der Epiphysenfuge respektiert wird.
- Dislozierte Eminentiaausrisse sollten operativ fixiert werden, hier zeigen die Techniken der Miniarthrotomie und das rein arthroskopische Verfahren längerfristig gleich gute $\mathrm{Er}$ gebnisse.

- Die Nachbehandlung bei Kindern sollte in jedem Fall in einer kindgerechten Orthese erfolgen, um das operative Ergebnis langfristig zu sichern.

\section{Korrespondenzadresse}

\section{Dr. T. Lein}

Klinik für Unfallchirurgie

Diakonissenkrankenhaus Dresden

Holzhofgasse 29, 01099 Dresden

thomas.lein@diako-dresden.de

\section{Einhaltung ethischer Richtlinien}

Interessenkonflikt. T. Lein gibt an, dass kein Interessenkonflikt besteht.

Dieser Beitrag beinhaltet keine Studien an Menschen oder Tieren.

The supplement containing this article is not sponsored by industry.

\section{Literatur}

1. Barrack RL, Bruckner JD, Kneisl J et al (1990) The outcome of nonopereratively treated complete tears of the anterior cruciate ligament in active young adults. Clin Orthop Relat Res 259:192-199

2. Frosch KH, Preiss A (2011) Knieverletzungen im Wachstumsalter. Trauma Berufskrankh 15(Suppl 1):52-58

3. Frosch KH, Stengel D, Brodhun T et al (2010) Outcomes and risks of the treatment of rupture of the anterior cruciate ligament in children and adolescents. Arthroscopy 26:1539-1550

4. Kannus P, Jarvinen M (1988) Knee ligament injuries in adolescents. Eight year follow-up of conservative management. J Bone Joint Surg Br 70:772776 
5. Kercher J, Xerogeanes J, Tannenbaum A, Al-Hakim R, Black JC, Zhao J (2009) Anterior cruciate ligament reconstruction in skeletally immature: an anatomical study utilizing 3-dimensional magnetic resonance imaging reconstructions. J Pediatr Orthop 29(2):124-129

6. Kocher MS, Saxon HS, Hovis WD et al (2002) Management and complications of anterior cruciate ligament injuries in skeletally immature patients: survey of the Herodicus Society and The ACL Study Group. J Pediatr Orthop 22:452-457

7. Louis ML, Guillaume JM, Launay F, Toth C, Jouvre $J$, Bollini G (2008) Surgical management of type II tibial intercondylar eminence fractures in children. J Pediatr Orthop B 17:231-235

8. Lukas C, Eberhardt O, Wirth T et al (2007) Results of $\mathrm{ACL}$ reconstruction with aperiost-patella tendon-periost graft in growth age. Z Orthop Unfall 145:706-711

9. Mäkelä EA, Vainonpäa S, Vihtonen K, Mero M, Roikkanen P (1988) The effect of trauma to the lower femoral epiphyseal plate. An experimental study in rabbits. J Bone Joint Surg Br 70:187-191

10. Mauch F, Parsch K (2004) Kindliche Ausrissverletzung der Eminentia intercondylaris. Orthop Traumatol 16:418-432

11. Meyers MH, McKeefer FM (1959) Fracture of the intercondylar eminence of the tibia. J Bone Joint Surg Am 41:209-220

12. Millett PJ, Willis AA, Warren RF (2002) Associated injuries in pediatric and adolescent anterior cruciate ligament tears: does a delay in treatment increase the risk of meniscal tear? Arthroscopy 18:955959

13. Mow VC, Hulskes R (1991) Structure and functions of tendons and ligaments. In: Woo SL, Young EP (eds) Basic orthopaedic biomechanics \& mechanobiology. Lippincott-Raven, Philadelphia, pp 220227

14. Mylle J, Renders P, Broos P (1993) Transepiphyseal fixation of anterior cruciate avulsion in a child: report of a complication and review of the literature. Arch Orthop Trauma Surg 112:101-103

15. Nordentoft EL (1969) Experimental epiphyseal in: grading of traumas and attempts at treating traumatic epiphyseal arrest in animals. Acta Orthop Scand 40:176-192

16. Osti M, Benedetto KP (2010) Arthroskopisch assistierte Refixation von Frakturen der Eminentia intercondylaris. Arthroskopie 23:92-96

17. Owens BD, Crane GK, Plante T, Busconi BD (2003) Treatment of type III tibial intercondylar eminence fractures in skeletally immature athletes. Am J Orthop 32:103-105

18. Pape D, Griffin R (2005) Arthroscopic endobutton fixation for tibial eminence fractures: surgical technique. J Knee Surg 18:203-205

19. Pous JG, Baldet P, Dimeglio A, Bonnel F (1979) Les traumatismes du cartilage de conjugaison. Notions fondamentales. Rev Chir Orthop Reparatrice Appar Mot 65(5):259-265

20. Preiss A, Brodhun T, Stietencron I, Frosch KH (2012) Die vordere Kreuzbandruptur im Wachstumsalter - operativ oder konservativ? Ein systematisches Review. Unfallchirurg 112:848-854

21. Seil R, Kohn D (2000) Ruptures of the anterior cruciate ligament $(\mathrm{ACL})$ during growth. Bull Soc Sci Med Grand Duche Luxemb 1:39-53

22. Seil R, Robert H (2004) Complete anterior cruciate ligament tears in children. Rev Chir Orthop Reparatrice Appar Mot 90(3):11-20
23. Seon JK, Song EK, Yoon TR (2005) Transphyseal reconstruction of the anterior cruciate ligament using hamstring autograft in skeletally immature adolescents. Korean Med Sci 20:1034-1038

24. Shelbourne KD, Patel DV, McCarroll JR (1996) Management of anterior cruciate ligament injuries in skeletally immature adolescents. Knee Surg Sports Traumatol Arthrosc 4:68-74

25. Sommerfeld DW (2008) Die arthroskopisch assistierte Refixation bei knöchernen Ausrissen des vorderen Kreuzbandes im Kindes- und Jugendalter. Oper Orthop Traumatol 20:310-320

26. Stanitski CL, Harvell JC, Fu F (1993) Observations on acute knee hemarthrosis in children and adolescents. J Paediatr Orthop 13:506-510

27. Strobel MW (2005) Vordere Kreuzbandinsuffizienz. In: Wirth CJ, Zichner L, Kohn D (Hrsg) Orthopädie und orthopädische Chirurgie. Thieme, Stuttgart, S 263-288

28. Ulmer M, Mehling AP, Jäger A (2008) Kindliche vordere Kreuzbandruptur. Arthroskopie 21:279-282

29. Welsch F, Mehling AP, Stein T, Hoffmann R (2013) Ersatz des vorderen Kreuzbandes beim Kind. Bereits Standard? Trauma Berufskrankh 15(Suppl 2):204-211

30. Zaricznyj B (1977) Avulsion fracture of the tibial eminence: treatment by open reduction and pinning. J Bone Joint Surg Am 59:1111-1114 